\title{
Effects of diltiazem on perioperative ischemia, arrhythmias, and myocardial function in patients undergoing elective coronary bypass grafting
}

A prospective, randomized study was performed on 120 patients undergoing elective coronary bypass grafting to define the effect of the calcium channel blocker diltiazem on perioperative ischemia, arrhythmias, and myocardial function. Patients received a continuous 24-hour perioperative infusion of either diltiazem $(0.1 \mathrm{mg} / \mathrm{kg}$ per hour, $n=60)$ or nitroglycerin $(1 \mu \mathrm{g} / \mathrm{kg}$ per minute, $n=60)$. Perioperative monitoring included hemodynamic measurements, three-channel Holter monitoring, repeated assessment of 12-lead electrocardiograms, and analysis of ischemia-specific laboratory parameters (creatine kinase, creatine kinase-MB, and creatine kinase-MB-mass and troponin-T). Global and regional systolic function and diastolic compliance were assessed by means of transesophageal echocardiography. The two groups did not differ with respect to preoperative and operative data. Except for a significant reduction in perioperative heart rate, diltiazem had no influence on hemodynamic parameters. The number $(17 \pm 9$ versus $25 \pm 5, p<0.05)$ and the duration $(69 \pm 47$ versus $104 \pm 87$ minutes, $p<0.05$ ) of transient ischemic events were significantly reduced as compared with the nitroglycerin group. In addition, peak values of all assessed laboratory parameters except creatine kinase were significantly lower in the diltiazem group. Patients treated with diltiazem had a lower incidence of perioperative atrial fibrillation $(5 \%$ versus $18 \%, p<0.05)$ and lower numbers of ventricular premature beats per hour $(10 \pm 8$ versus $19 \pm 22, p<0.05)$ and ventricular runs per hour $(5 \pm 17$ versus $32 \pm 38, p<0.05)$. Postoperatively, the percent fractional area of contraction and percent systolic wall thickening of the anterior wall were significantly improved in the diltiazem group but not in the nitroglycerin group. In addition, the postoperative diastolic flow/velocity ratio was significantly lower in the nitroglycerin group than in the diltiazem group $(0.949 \pm 0.391$ versus $1.331 \pm 0.475, p<0.001)$. It is concluded that perioperative infusion of the calcium antagonist diltiazem has no adverse effect on perioperative hemodynamics and systolic myocardial function and provides potent antiischemic and antiarrhythmic protection in patients undergoing coronary bypass grafting. (J ThORAC CardiovasC SURG 1994;107:811-21)

Rainald Seitelberger, MD (by invitation), Waltraud Hannes, MD (by invitation),

Mark Gleichauf, MD (by invitation), Matthias Keilich, BS (by invitation),

Marion Christoph, BS (by invitation), and Roland Fasol, MD (by invitation),

Freiburg, Germany

Sponsored by Ernst Wolner, MD, Vienna, Austria

From the Department of Cardiovascular Surgery, University of Freiburg, Freiburg, Germany

Read at the Seventy-third Annual Meeting of The American Association for Thoracic Surgery, Chicago, Ill., April 25-28, 1993.

Address for reprints: Rainald Seitelberger, MD, II. Chirurgische Universitätsklinik, Spitalgasse 23, A-1090 Vienna, Austria.

Copyright $\odot 1994$ by Mosby-Year Book, Inc.

$0022-5223 / 94 \$ 3.00+0 \quad \mathbf{1 2 / 6 / 5 2 6 1 6}$

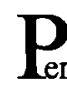
Perative mortality and morbidity in patients undergoing elective coronary bypass procedures (CABG) have remained relatively stable at a remarkably low level during recent years. ${ }^{1-3}$ Nevertheless, increasing numbers of patients with more severe coronary artery disease and impaired left ventricular function demand further improvements in perioperative patient management. Various forms of intraoperative myocardial protection 
such as systemic and local hypothermia, antegrade and retrograde hypothermic crystalloid or blood cardioplegia, and warm blood cardioplegia have been implicated during recent years. The quality of intraoperative protection certainly contributes to the preservation of the functional integrity of the myocardium during the ischemic period. However, its influence on the prevalence of myocardial ischemia and arrhythmias during the reperfusion and early postoperative periods remains questionable. Consequently, adequate perioperative myocardial protection should include not only the intraoperative but also the early postoperative period.

We have shown that the perioperative infusion of calcium channel antagonists such as nifedipine $e^{4,5}$ and diltiazem ${ }^{6}$ up to 24 hours after the operation substantially decreases the prevalence and extent of postoperative myocardial ischemia. In addition, diltiazem also proved effective in the prevention of postoperative supraventricular and intraventricular arrhythmias. However, there is no detailed information on the influence of the perioperative infusion of calcium antagonists on the preservation of perioperative myocardial function. Diltiazem is known to exert negative inotropic effects under various conditions ${ }^{7,8}$ and may have a deleterious effect on postoperative functional recovery, especially in patients with impaired ventricular contractility.

The present study was undertaken to clarify the role of diltiazem as an effective therapeutic measurement for perioperative protection in patients undergoing $\mathrm{CABG}$. In addition to close monitoring of perioperative ischemia and arrhythmias by electrocardiograms, Holter recordings, and measurement of ischemia-specific laboratory parameters, perioperative changes in systolic and diastolic myocardial function were assessed by transesophageal echocardiography. In this randomized trial, patients treated with diltiazem were compared with a control group receiving standard perioperative protection with nitroglycerin.

\section{Patients and methods}

The study was performed on 120 patients undergoing elective CABG. Patients with unstable angina, preoperative left or right bundle branch block, additional surgical or redo procedures, or a rethoracotomy made necessary by excessive postoperative bleeding were excluded from the study. The study protocol was approved by the Ethics Committee on Human Research of the University of Freiburg. Written informed consent was obtained from each patient. The patients were randomly assigned to receive nitroglycerin $(n=60)$ or diltiazem $(n=60)$. Routine medication (nitrates, $\beta$-blockers, or calcium antagonists) was terminated the evening before the operation.

All surgical procedures for which saphenous vein grafts or internal mammary artery grafts, or both, were used were performed under moderate hypothermia at $31^{\circ} \mathrm{C}$ with a mem- brane oxygenator with linear flow. For myocardial protection during cardiac arrest, cold potassium cardioplegic solution $\left(1000 \mathrm{ml}\right.$ containing $82.5 \mathrm{mmol} \mathrm{Na}{ }^{++}, 30 \mathrm{nmol} \mathrm{K}^{+}, 0.5 \mathrm{mmol}$ $\mathrm{Ca}^{++}, 5$ gm glucose, 10 gm mannitol, and $26.8 \mathrm{ml} 8.4 \%$ sodium bicarbonate) was given via the aortic root in addition to topical cooling by saline slush. Body rewarming began during completion of the last distal anastomosis. A partial occlusion clamp was used for the proximal anastomoses.

Patients assigned to the diltiazem group had continuous diltiazem infusion (minimum dose $0.1 \mathrm{mg} / \mathrm{kg}$ per hour) from the onset of extracorporeal circulation until 24 hours after the aortic crossclamp time. During the same period of time, patients assigned to the control group had continuous nitroglycerin infusion (minimum dose $1 \mu \mathrm{g} / \mathrm{kg}$ per minute). The infusion rate was increased in both groups in cases of postoperative hypertension (>90 mm Hg mean arterial blood pressure).

Hemodynamic measurements. Hemodynamic parameters were assessed after the operation and at $1,2,4,8,12,16,20$, and 24 hours after aortic crossclamp removal. Heart rate, mean arterial pressure, mean pulmonary artery pressure, pulmonary capillary wedge pressure, and central venous pressure were recorded by an eight-channel recorder (Siemens system sirecust, model 1280; Siemens AG, Fuerth, Germany) and transducers (Siemens, model 1280). An Edwards Laboratory cardiac output computer (model 9520; Baxter Healthcare Corp., Edwards Division, Irvine, Calif.) was used to perform cardiac output determinations with the thermodilution technique. From these data, cardiac index and pulse-pressure rate were calculated by standard formulas.

Electrocardiographic recordings and Holter monitoring. Twelve-lead electrocardiographic recordings were performed shortly before the operation and $2,4,8,12,16,20,24,48,72$, and 96 hours after aortic crossclamp removal. Continuous three-channel Holter monitoring was performed in the postoperative period with Zymed Holter recorders (Zymed, Inc., Camarillo, Calif.). The 24-hour monitoring period was started 2 hours after aortic crossclamp removal in the intensive care unit. The electrodes were placed so that channels 1 to 3 approximated electrocardiographic leads $V_{2}, V_{5}$, and II, respectively. All tapes were analyzed for arrhythmias and ST-segment alterations on a semiautomatic basis (Zymed Quickpage Holter device, system 1210) by the same investigator, blinded to the patient's group assignment.

Rhythm analysis included the prevalence of atrial fibrillation and atrioventricular block and the incidence of ventricular arrhythmias per hour, defined as ventricular premature complexes (VPCs, two ventricular premature beats) and ventricular runs (VRs, three or more ventricular premature complexes in succession at a rate between 100 and 200 cycles $/ \mathrm{min}$ ).

Two different forms of perioperative myocardial ischemia were defined by the combined analysis of electrocardiogram and Holter recordings according to the following criteria:

Transient ischemic event. A transient ischemic event is defined as horizontal or down-sloping ST-segment depression of $1 \mathrm{~mm}$ or more lasting at least 1 minute and measured 60 to 80 msec beyond the $J$ point of the QRS complex in at least one Holter channel with no signs of evolving myocardial infarction.

Myocardial infarction. A myocardial infarction is defined as persistent typical ST-segment elevation of $2 \mathrm{~mm}$ or more measured 60 to $80 \mathrm{msec}$ from the $\mathrm{J}$ point in at least one Holter channel and development of a new $Q$ wave $(>0.04$ second in duration and more than one fourth of the following $R$ wave in 
Table I. Preoperative clinical data

\begin{tabular}{lcc}
\hline \multicolumn{1}{c}{ Variable } & Diltiazem & Nitroglycerin \\
\hline Patients $(n)$ & 60 & 60 \\
Male (\%) & $46(76.6)$ & $48(80.0)$ \\
Female (\%) & $14(23.4)$ & $12(20.0)$ \\
Age (yr) & $62.3 \pm 8$ & $62.6 \pm 8$ \\
Two-vessel disease (\%) & $11(18.4)$ & $13(21.6)$ \\
Three-vessel disease (\%) & $49(81.6)$ & $47(78.4)$ \\
Left main stenosis (\%) & $14(23.3)$ & $15(25.0)$ \\
Preoperative MI (\%) & $42(70.0)$ & $43(71.6)$ \\
NYHA functional class (\%) & & \\
$\quad$ I-II & $10(16.6)$ & $5(8.3)$ \\
II-III & $31(51.7)$ & $41(68.4)$ \\
III-IV & $19(31.7)$ & $14(23.3)$ \\
Preoperative therapy & & \\
$\quad$ Calcium antagonists (\%) & $25(41.6)$ & $29(48.3)$ \\
$\beta$-blockers (\%) & $40(66.6)$ & $40(66.6)$ \\
\hline
\end{tabular}

$M I$, Myocardial infarction; $N Y H A$, New York Heart Association.

amplitude) in the corresponding 12-lead electrocardiograms, or persistent negative coronary $\mathrm{T}$ wave of more than $3 \mathrm{~mm}$ in 12-lead electrocardiograms during the 96-hour postoperative observation period without the occurrence of a new $Q$ wave.

Biochemical analysis. In all patients, serum levels of creatine kinase ( $\mathrm{CK}$ ), $\mathrm{MB}$ isoenzyme activity (CK-MB, enzymatic fluorometric methods, Boehringer Mannheim GmbH, Mannheim, Germany), CK-MB-mass concentration analysis (CKMB-M, Novo Clone CK-MB mass concentration assay, Novo Industri $A / S$, New York, N.Y.), and troponin-T (enzymelinked immunosorbent assay of troponin- $T$, Boehringer Mannheim) were measured before the operation and 1, 2, 4, 8, 12, 16, $20,24,48,72$, and 96 hours after opening of the aortic crossclamp.

Transesophageal echocardiography. Transesophageal echocardiography was assessed preoperatively after induction of anesthesia and 4 hours after cardiopulmonary bypass. The echocardiographic images were obtained with a $5.0 \mathrm{MHz}$ faced-array transducer (Hewlett-Packard HP 21362 C; Hewlett-Packard Co., Palo Alto, Calif.) mounted to an echo scope interfaced to an ultrasound unit (Hewlett Packard HP SONOS 1000 [77020 AC]). Images were recorded on videotape in real-time and stop-frame format. So that systolic myocardial function could be assessed, the transgastric short-axis view of the left ventricle at the mid-papillary level was achieved. The cross-sectional left ventricular image was subdivided into four segments: anterior, lateral, posterior, and interventricular septum. Wall motion was qualitatively graded for each segment as normal, hypokinetic, or severely hypokinetic-akinetic.

For quantitative assessment the off-line end-diastolic and end-systolic boundaries of the left ventricle were traced with a Siemens AVD system. The R wave was used to select end-diastolic frames; end-systole was defined as the minimal cross section of the left ventricle. From these data, the fractional area of contraction (\%FAC) of the left ventricle was calculated from the difference of the end-diastolic area (EDa) and the endsystolic area (ESa) related to the end-diastolic area: $(\mathrm{EDa}-\mathrm{ESa}) / \mathrm{EDa} \times 100$. For assessment of changes in regional wall motion, end-systolic (ES) and end-diastolic (ED) boundaries of the interventricular septum and the anterior, lat-
Table II. Surgical data

\begin{tabular}{lcc}
\hline \multicolumn{1}{c}{ Variable } & Diltiazem & Nitroglycerin \\
\hline Patients $(n)$ & 60 & 60 \\
ACC time (min) & $61.2 \pm 23.9$ & $62.7 \pm 26.7$ \\
ECC (min) & $99.6 \pm 34.6$ & $100.2 \pm 35.3$ \\
Grafts/patient $(n)$ & $3.5 \pm 0.7$ & $3.5 \pm 0.7$ \\
Complete revascularization (\%) & $56(93.3)$ & $54(90.0)$ \\
IMA grafts (\%) & $43(71.6)$ & $45(75.0)$ \\
Mortality (\%) & $1(1.6)$ & 0 \\
\hline
\end{tabular}

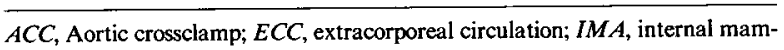
mary artery.

eral, and posterior walls of the left ventricle were traced and percent systolic wall thickening was calculated by the formula: (ES - ED) $/ \mathrm{ES} \times 100$.

The diastolic function of the left ventricle was assessed in the four-chamber view. The sample volume was placed between the tips of the mitral leaflets, where the velocity time integrals were obtained. Diastolic flow velocity waveforms from three cardiac cycles were characterized quantitatively and averaged. The velocity time integrals of the early (vTI E) and late (vTI A) diastolic filling were assessed and the diastolic flow/velocity ratio ( $E / A$ ratio) as an indicator of left ventricular diastolic compliance was calculated by the formula: vTI E/vTI A.

Statistical analysis. Results were expressed as mean \pm standard deviation of the mean. Standard $\chi^{2}$ analysis was used for comparison of baseline categorical (anumeric) factors. The unpaired, two-tailed Student's $t$ test was used to compare groups of data. In addition, the Wilcoxon rank sum test was performed for each parameter. Analysis of variance was used to compare the repeated hemodynamic data with diltiazem versus nitroglycerin as the grouping factor and time as the within-group factor. For all tests the level of significance was set at $5 \%$ of confidence $(p<0.05)$.

\section{Results}

Tables I and II show preoperative clinical and surgical data of all patients. There were no significant differences between the two groups concerning the preoperative clinical data (Table I) such as severity of coronary artery disease, New York Heart Association classification, and therapeutic regimen and surgical data (Table II), such as aortic crossclamp time, bypass time, number of distal anastomoses, and number of internal mammary artery grafts.

Whereas perioperative mortality in the nitroglycerin group was $0 \%$, one patient $(1.6 \%)$ in the diltiazem group died 22 days after the operation of postoperative pneumonia, septicemia, and subsequent multiorgan failure. Postmortem autopsy revealed patent grafts and no signs of recent myocardial infarction. Because all study-related data were assessed during the first 6 days after the operation, the data of this patient were included in the analysis.

None of the patients had inotropic medication during 
Table III. Hemodynamic data $(\mathrm{n}=60$ in each group)

\begin{tabular}{|c|c|c|c|c|c|c|c|c|c|}
\hline Variable & Preop. & $1 \mathrm{hr}$ & $2 h r$ & $4 h r$ & $8 h r$ & $12 \mathrm{hr}$ & $16 \mathrm{hr}$ & $20 \mathrm{hr}$ & $24 \mathrm{hr}$ \\
\hline \multicolumn{10}{|c|}{ Heart rate (beats $\cdot \min ^{-1}$ ) } \\
\hline D & $59.9 \pm 9.6$ & $77.5 \pm 14.2$ & $72.9 \pm 13.4^{*}$ & $83.2 \pm 14.9^{*}$ & $80.4 \pm 13.5 \dagger$ & $81.8 \pm 13.7^{*}$ & $83.1 \pm 14.7^{*}$ & $81.6 \pm 12.8 \dagger$ & $81.0 \pm 12.8 \dagger$ \\
\hline $\mathrm{N}$ & $61.0 \pm 12.3$ & $82.0 \pm 13.2$ & $80.4 \pm 13.3$ & $89.7 \pm 15.1$ & $90.1 \pm 13.4$ & $88.4 \pm 14.5$ & $88.9 \pm 14.3$ & $88.1 \pm$ & $88.2 \pm 14.5$ \\
\hline \multicolumn{10}{|c|}{ Mean arterial pressure $(\mathrm{mm} \mathrm{Hg})$} \\
\hline $\mathrm{D}$ & $77.9 \pm 9.9$ & $76.7 \pm 9.9$ & $84.6 \pm 16.1$ & $80.9 \pm 13.6$ & $72.9 \pm$ & 70.8 & $70.7 \pm 9.7$ & $74.4 \pm 11.0$ & $75.9 \pm 11.4$ \\
\hline $\mathbf{N}$ & $74.1 \pm 11.2$ & $75.8 \pm 10.6$ & $83.6 \pm$ & $78.7 \pm$ & $75.2 \pm$ & 72.4 & $72.9 \pm$ & 72.9 & 74.9 \\
\hline \multicolumn{10}{|c|}{ Pulmonary capillary wedge pressure $(\mathrm{mm} \mathbf{H g}$ ) } \\
\hline D & $9.6 \pm 3.4$ & $10.1 \pm 2.7$ & $10.5 \pm 3.5$ & & 10 & 9.9 & $10.3 \pm 4.0$ & $11.1=$ & $11.6 \pm 4.2$ \\
\hline $\mathbf{N}$ & $9.3 \pm$ & $10.7 \pm 3.8$ & $10.9 \pm 4.3$ & 11. & 10.9 & 10.5 & 9.9 & $10.6=$ & $11.3 \pm 5.1$ \\
\hline \multicolumn{10}{|c|}{ Mean pulmonary artery pressure $(\mathrm{mm} \mathrm{Hg}$ ) } \\
\hline D & $15.2 \pm 3.9$ & $16.7 \pm 3.7$ & $18.2 \pm 4.8$ & & 2 & 18.3 & $17.7 \pm 4.8$ & $18.7 \pm 5.4$ & $18.6 \pm 4.8$ \\
\hline $\mathbf{N}$ & $15.1 \pm 4.8$ & $17.1 \pm 5.0$ & $18.8 \pm 5.5$ & 19.7 & $19.5 \pm 4.6$ & 19.3 & $17.5 \pm 4.8$ & $18.2 \pm$ & $18.8 \pm 6.0$ \\
\hline \multicolumn{10}{|c|}{ Cardiac index $\left(\mathrm{L} \cdot \mathrm{min}^{-1} \cdot \mathrm{m}^{-2}\right)$} \\
\hline D & $2.0 \pm 0.4$ & $2.4 \pm 0.5$ & $2.3 \pm$ & $2.9 \pm$ & 3.1 & $3.2 \pm 0.7$ & $3.1 \pm 0.7$ & $3.0 \pm 0.7$ & $3.0 \pm 0.7$ \\
\hline $\mathbf{N}$ & $2.0 \pm 0.4$ & $2.5 \pm 0.6$ & $2.4 \pm 0.5$ & $2.9 \pm 0.8$ & $3.1 \pm 0.7$ & 3.1 & $3.1 \pm 0.6$ & $3.0 \pm 0.6$ & $3.0 \pm 0.5$ \\
\hline \multicolumn{5}{|c|}{ Pulse pressure rate $\left(\mathrm{mm} \mathrm{Hg} \cdot\right.$ beats $\left.\cdot \min ^{-1} \cdot 10^{-3}\right)$} & . & & & & \\
\hline D & $6.25 \pm 1.1$ & $7.81 \pm 1.4^{*}$ & $8.80 \pm 2.6^{*}$ & $10.21 \pm 2.4^{*}$ & $9.11 \pm 2.1^{*}$ & $8.65 \pm 2.5^{*}$ & $8.51 \pm 2.2^{*}$ & $9.13 \pm 1.8 \dagger$ & $9.79 \pm 2.2 \dagger$ \\
\hline $\mathrm{N}$ & $6.47 \pm 1.9$ & $8.57 \pm 2.2$ & $9.67 \pm 2.4$ & $11.50 \pm 2.1$ & $10.41 \pm 1.4$ & $9.84 \pm 1.9$ & $9.75 \pm 1.9$ & $10.51 \pm 2.0$ & $11.02 \pm 2.1$ \\
\hline
\end{tabular}

Hemodynamic parameters before and for 24 hours after aortic crossclamp removal. $D$, Diltiazem group; $N$, nitroglycerin group.

*Value differs significantly from Nitroglycerin group $(p<0.05)$.

$\nmid$ Value differs significantly from Nitroglycerin group $(p<0.01)$.

the period of preoperative data acquisition. Postoperatively, eight patients in the diltiazem group (13\%) and seven patients in the nitroglycerin group $\left(12 \%, p=\mathrm{NS}^{*}\right)$ required temporary inotropic drug support (low-dose dobutamine) for hemodynamic stabilization. In all of these patients, the dobutamine infusion could be terminated between 2 and 16 hours after cardiopulmonary bypass. At the time of postoperative functional assessment by transesophageal echocardiography ( 4 hours after cardiopulmonary bypass), inotropic drug support was still performed in five patients of the diltiazem group ( $8 \%$ ) and in five patients of the nitroglycerin group (8\%).

Hemodynamics. Table III depicts preoperative and postoperative hemodynamic parameters for both groups. Throughout the 4- to 24-hour period after aortic crossclamp removal, heart rate and the calculated pulse-pressure rate as an indicator of myocardial oxygen demand were significantly lower in the diltiazem group. The other hemodynamic parameters did not differ between the two treatment groups.

Supraventricular and ventricular arrhythmias. Table IV summarizes supraventricular and ventricular arrhythmias and conduction abnormalities for both groups. Whereas postoperative atrial fibrillation was substantially less prevalent in the diltiazem group, the prevalence of first-degree atrioventricular block was sim-

*NS $=$ Not significant. ilar in both groups. In all cases the occurrence of atrioventricular block was transient and none of the patients in either group required therapeutic interventions. There was no case of postoperative ventricular fibrillation or ventricular tachycardia ( $>10$ consecutive VPCs). The prevalence of postoperative VPCs, VPCs $>30$ /hour and VRs did not significantly differ between groups. However, patients treated with diltiazem had significantly fewer VPCs, VPCs $>30$ /hour, and VPCs/ hour during periods of ST-segment depression and VRs/ hour. In addition, patients with postoperative arrhythmias classified as Lown II (VPCs >30/hour) had significantly fewer VPCs/hour when treated with diltiazem.

Myocardial ischemia and laboratory parameters. Data on perioperative myocardial ischemia are depicted in Table V. Perioperative myocardial infarction and transient ischemic events were less prevalent in the diltiazem group. However, the differences did not reach statistical significance. The number of events per patient and the average duration of events were substantially higher in the nitroglycerin group than in the diltiazem group.

Peak values of ischemia-specific laboratory parameters are listed in Table VI. Peak values of CK-MB, CK-MB$M$, and troponin- $T$ were significantly lower in the diltiazem group than in the nitroglycerin group when calculated for all patients. However, there was no statistically 
Table IV. Postoperative arrhythmias

\begin{tabular}{lcc}
\hline \multicolumn{1}{c}{ Variable } & Diltiazem & Nitroglycerin \\
\hline Atrial fibrillation (\%) & $3(5.0)$ & $11(18.3)^{*}$ \\
First-degree atrioventricular block $(\%)$ & $3(5.0)$ & $4(6.7)$ \\
VPCs (\%) & $57(95)$ & $52(87)$ \\
VPCs $/ \mathrm{hr}$ & $10 \pm 8$ & $19 \pm 22^{*}$ \\
VPCs $>30 / \mathrm{hr}(\%)$ & $5(8.3)$ & $10(16.6)$ \\
VPCs $>30 / \mathrm{hr}$ & $58 \pm 26$ & $188 \pm 68^{*}$ \\
VPCs during ST depression $/ \mathrm{hr}$ & $1.4 \pm 0.6$ & $2.8 \pm 0.9^{*}$ \\
VRs $(\%)$ & $57(95.0)$ & $52(86.7)$ \\
VRs $/ \mathrm{hr}$ & $5 \pm 17$ & $32 \pm 38^{*}$ \\
\hline
\end{tabular}

*Value differs significantly from nitroglycerin group $(p<0.05)$.

Table V. Perioperative ischemia

\begin{tabular}{lcc}
\hline \multicolumn{1}{c}{ Variable } & Diltiazem & Nitroglycerin \\
\hline MI (\%) & $2(3.3)$ & $4(6.7)$ \\
TIE $(\%)$ & $15(25.0)$ & $19(31.6)$ \\
Events $(n)$ & $17.6 \pm 9.1$ & $25.3 \pm 5.4^{*}$ \\
Duration (min) & $69.7 \pm 47.4$ & $104.8 \pm 87.6^{*}$
\end{tabular}

$M I$, Myocardial infarction; TIE, transient ischemic event.

$*$ Value differs significantly from nitroglycerin group $(p<0.05)$.

significant difference between the CK peak values of the two groups. Patients with transient ischemic events had significantly lower troponin- $T$ peak values when treated with diltiazem. In this subgroup of patients, the differences in peak values for CK, CK-MB, and CK-MB-M were not statistically significant between the two treatment groups. In patients with perioperative myocardial infarction, however, troponin-T and CK-MB-M were significantly lower in the diltiazem group.

Transesophageal echocardiography. Qualitative assessment of regional wall motion showed equal preoperative distribution of normokinetic, hypokinetic, and severely hypokinetic-akinetic segments in both treatment groups. Four hours after the operation, there was a tendency toward more normokinetic segments in both groups. Patients treated with diltiazem had fewer severely hypokinetic segments after the operation, whereas almost no change was observed in the nitroglycerin group (Fig. $1 ; p=\mathrm{NS}$ ). With regard to postoperative grading changes in preoperatively normokinetic, hypokinetic, or severely hypokinetic-akinetic segments, no significant differences were detected between the groups (Fig. 2). However, especially in segments that were severely hypokinetic-akinetic before the operation, infusion of diltiazem appeared to induce more pronounced improvement in functional grading as indicated by the lower number of postoperative akinetic segments ( $40.5 \%$ versus $50 \%$ in the nitroglycerin group, $p=\mathrm{NS}$ ).

The global systolic function of the left ventricle,
Table VI. Enzyme data

\begin{tabular}{lccc}
\hline \multicolumn{1}{c}{ All patients: } & $\begin{array}{c}\text { Patients } \\
\text { with TIE: }\end{array}$ & $\begin{array}{c}\text { Patients } \\
\text { with MI: }\end{array}$ \\
& $D(\mathrm{n}=60)$ & $D(\mathrm{n}=15) ;$ & $D(\mathrm{n}=2) ;$ \\
Variable & $N(\mathrm{n}=60)$ & $N(\mathrm{n}=19)$ & $N(\mathrm{n}=4)$ \\
\hline CK $(\mathrm{U} / \mathrm{L})$ & & & \\
D & $531.1 \pm 429$ & $415.6 \pm 348.5$ & $1034.8 \pm 682$ \\
N & $545.2 \pm 353$ & $546.0 \pm 334.7$ & $734.8 \pm 334$ \\
CK-MB $(\mathrm{U} / \mathrm{L})$ & & \\
D & $17.6 \pm 14.3^{*}$ & $17.5 \pm 9.2$ & $45.8 \pm 28.3^{*}$ \\
N & $25.3 \pm 16.3$ & $24.9 \pm 13.0$ & $64.3 \pm 33.3$ \\
MB-M $(\mu \mathrm{g} / \mathrm{L})$ & & \\
D & $35.2 \pm 38.4^{*}$ & $31.6 \pm 22.1$ & $109.4 \pm 97.5^{*}$ \\
N & $51.5 \pm 33.9$ & $51.7 \pm 34.3$ & $196.2 \pm 135.4$ \\
Troponin-T $(\mu \mathrm{g} / \mathrm{L})$ & & \\
D & $0.98 \pm 0.7^{*}$ & $1.20 \pm 0.6^{*}$ & $3.47 \pm 1.4^{*}$ \\
N & $1.70 \pm 0.8$ & $1.97 \pm 0.5$ & $6.05 \pm 1.9$ \\
\hline
\end{tabular}

$D$, Diltiazem group; $N$, nitroglycerin group; $T I E$, transient ischemic event; $M I$, myocardial infarction; $C K$, creatine kinase; $C K-M B, M B$ isoenzyme of $\mathrm{CK}$; $M B-M$, creatine kinase MB mass.

*Value differs significantly from nitroglycerin group $(p<0.05)$.

expressed as percent fractional area of contraction (\%FAC), was higher in both groups 4 hours after the operation than before the operation (Table VII). However, the difference reached statistical significance only in the diltiazem group. In addition, percent systolic wall thickening, calculated as a quantitative parameter for changes in regional myocardial function, significantly increased in the anterior wall in patients treated with diltiazem. Percent systolic thickness of the lateral and posterior walls as well as the interventricular septum, however, was not significantly improved at postoperative evaluation. In the nitroglycerin group, none of the assessed wall segments was improved in comparison with preoperative measurements. Percent systolic thickness in the posterior wall was even slightly but significantly decreased at postoperative evaluation (Table VII).

With regard to the diastolic compliance of the left ventricle, both treatment groups had almost identical preoperative flow/velocity ratios (Fig. 3). The flow/velocity ratio was significantly reduced in both groups 4 hours after the operation. However, the decrease was markedly lower in the diltiazem group than in the nitroglycerin group, indicating a superior effect of diltiazem on postoperative diastolic compliance.

There were no correlations between prevalence and numbers of postoperative ventricular arrhythmias and preoperative ventricular function (\%FAC), regardless of whether patients were treated with diltiazem or nitroglycerin. Even in patients with more severe combinations of ventricular arrhythmias (VPCs >30/hour and VRs), the preoperative $\mathrm{FAC} \%$ was similar (diltiazem group, 
$45.7 \pm 9$; nitroglycerin group, $51.1 \pm 10$ ) when compared with that of patients without both types of arrhythmias during the postoperative observation period (diltiazem group, $46.6 \pm 9$; nitroglycerin group, $46.0 \pm 10$ ). In addition, because of the high percentage of patients with complete revascularization in both groups (see Table II), the absence of complete revascularization was not significantly correlated with a greater prevalence of postoperative arrhythmias, higher levels of ischemia-specific laboratory parameters, or depressed postoperative ventricular function.

\section{Discussion}

Perioperative infusion of antiischemic and antiarrhythmic substances appears to be remarkably effective in improving myocardial protection during and early after CABG. We have previously demonstrated a significant reduction in prevalence and extent of perioperative myocardial ischemia by perioperative infusion of nifedipine, with and without additional application of the $\beta$-blocker metoprolol, or diltiazem. ${ }^{4-6,9}$ Diltiazem also proved to be an effective antiarrhythmic substance in the perioperative setting.

The antiischemic efficacy of diltiazem in experimental and clinical manifestations of coronary artery disease is widely documented. ${ }^{7}{ }^{10-12}$ However, the negative inotropic effect may limit its application in CABG. Especially in patients with severe wall motion abnormalities, further depression of myocardial function may induce significant perioperative hemodynamic problems. Consequently, the present study focused not only on the antiischemic and antiarrhythmic efficacy of diltiazem but also on its effect on perioperative global and regional myocardial function. Our study clearly demonstrates that the antiischemic and antiarrhythmic efficacy of diltiazem is not accompanied by any detectable depressing effect on myocardial contractility.

Effects of diltiazem on systemic hemodynamic parameters. Diltiazem significantly reduced heart rate throughout the 24 postoperative hours. Because postoperative arterial blood pressure was comparable in the two groups, the pulse-pressure rate as an indicator of myocardial oxygen demand was also lower in the diltiazem group. However, although heart rate was significantly lower, cardiac index was comparable with that of nitroglycerin-treated patients, indicating that the negative inotropy of diltiazem did not significantly depress perioperative myocardial function. The effect of diltiazem in lowering heart rate may have contributed to its antiischemic efficacy, because an increased heart rate is a primary factor in the pathogenesis of myocardial ischemia. ${ }^{13,14}$
Effects of diltiazem on supraventricular and ventricular arrhythmias. In accordance with various experimental and clinical findings, diltiazem substantially reduced the prevalence of postoperative atrial fibrillation. ${ }^{15-18}$ The clinical importance of perioperative supraventricular arrhythmias and their contribution to the occurrence of postoperative ischemia or other complications appear to be insignificant. However, they are frequently not well tolerated by patients and induce various symptoms such as temporary hemodynamic instability, shortness of breath or chest discomfort. ${ }^{19}$ Consequently, the pharmacologic prevention of perioperative arrhythmias should be mandatory during postoperative intensive care management and is effectively provided by diltiazem.

Diltiazem is known to depress antegrade atrioventricular nodal conduction, ${ }^{7,20}$ which may lead to an increased need for perioperative external pacing. The low infusion rate of diltiazem in the present study, however, had no apparent influence on atrioventricular nodal conduction. The incidence of perioperative first-degree atrioventricular block was low and almost identical in both treatment groups ( $5 \%$ versus $6.7 \%$ ). No case of second- or third-degree atrioventricular block was observed. The conduction abnormality was not permanent in any of these patients and did not require temporary pacing to control hemodynamic instability. However, higher infusion rates of diltiazem may indeed lead to clinically significant conduction abnormalities.

Diltiazem did not reduce the overall prevalence of VPCs and VRs but significantly lowered the average number of VPCs and VRs per patient. Under normal conditions, diltiazem has only negligible effects on the effective refractory periods of the ventricle or His-Purkinje system. ${ }^{7}{ }^{15}$ However, it is known to suppress VPCs under ischemic conditions. This effect is due to an improvement of ischemia-induced prolongation and dispersion of conduction time. ${ }^{15,21}$

Effects of diltiazem on prevalence and extent of myocardial ischemia. The diagnosis of myocardial ischemia in the perioperative setting still provides significant problems. Most studies rely on the occurrence of a new $Q$ wave in postoperative electrocardiograms as an indicator for perioperative myocardial infarction. However, it was shown that this diagnostic approach is not only of limited sensitivity and specificity in cases of transmural infarction, but also misses the diagnosis of substantial myocardial ischemia and cell necrosis. ${ }^{22-24}$ In addition, because of its relatively low overall incidence in elective CABGs, the use of a new $Q$ wave as an end point diagnostic parameter for defining perioperative ischemia does not allow differentiation between the antiischemic efficacy of 

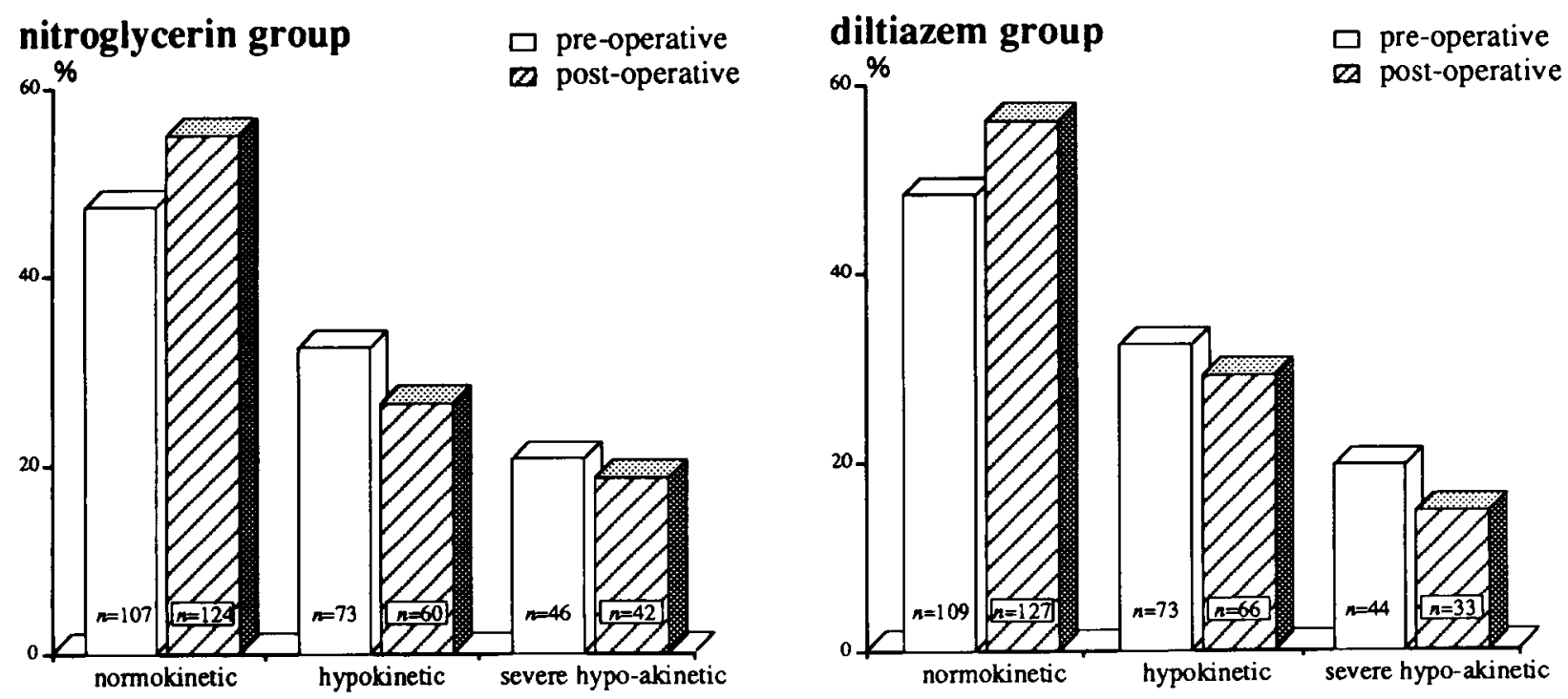

Fig. 1. Bar graph comparing qualitatively graded regional systolic myocardial function (normal, hypokinetic, or severe hypokinetic-akinetic) preoperatively and at 4 hours after crossclamp removal (postoperatively) in patients treated with either nitroglycerin or diltiazem. Data are given as percent from absolute values.

Table VII. Global and regional systolic function assessed by TEE

\begin{tabular}{|c|c|c|c|c|c|}
\hline & \multirow[b]{2}{*}{$\% F A C$} & \multicolumn{4}{|c|}{$\% W T h$} \\
\hline & & $A W$ & $L W$ & $P W$ & Septum \\
\hline \multicolumn{6}{|c|}{ Preoperative } \\
\hline D & $46.5 \pm 9.3$ & $26.8 \pm 9.5$ & $24.2 \pm 9.9$ & $22.2 \pm 8.9$ & $22.1 \pm 9.0$ \\
\hline $\mathbf{N}$ & $49.4 \pm 9.8$ & $25.5 \pm 10.7$ & $23.4 \pm 9.8$ & $22.9 \pm 9.1$ & $24.4 \pm 10.2$ \\
\hline \multicolumn{6}{|c|}{ Postoperative day 4} \\
\hline D & $51.7 \pm 10.2^{*}$ & $31.4 \pm 8.9^{*}$ & $26.0 \pm 8.5$ & $22.5 \pm 8.7$ & $22.5 \pm 8.3$ \\
\hline $\mathbf{N}$ & $51.1 \pm 9.8$ & $27.7 \pm 9.1$ & $26.6 \pm 8.9$ & $19.4 \pm 8.7^{*}$ & $23.6 \pm 9.9$ \\
\hline
\end{tabular}

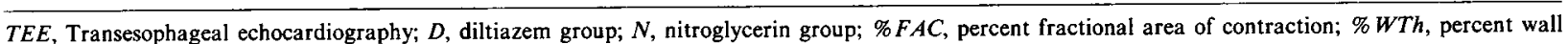
thickening; $A W$, anterior wall; $L W$, lateral wall; $P W$, posterior wall.

*Value differs significantly from preoperative $(p<0.05)$.

therapeutic regimens in studies with relatively low numbers of patients.

In the present study, myocardial ischemia was independently diagnosed by the combined analysis of serial 12-lead electrocardiograms and continuous Holter monitoring and the analysis of highly ischemia-specific laboratory parameters. In addition to the occurrence of a new $Q$ wave after persistent ST-segment elevation, persistent negative $T$ waves of more than $3 \mathrm{~mm}$ were also considered to be diagnostic of new perioperative myocardial infarction. Consequently, perioperative myocardial infarction was slightly more prevalent in the present investigation (3.3\% versus $6.7 \%)$ than in studies that rely solely on the occurrence of a new $Q$ wave as a proof for myocardial infarction. Nevertheless, we were unable to demonstrate a significant reduction in the prevalence of perioperative myocardial infarction by diltiazem, although infarction was only half as prevalent as that observed in the nitroglycerin group.

In addition to the occurrence of new myocardial infarction, the prevalence, number, and duration of transient ischemic events (ST-segment depression of $>1 \mathrm{~mm}$ ) were also included in the definition of perioperative ischemia. The results of this study demonstrate the prevalence of postoperative transient ischemic events (25\% in the diltiazem group versus $32 \%$ in the nitroglycerin group) with a substantial variability in frequency and duration. The lower incidence of transient ischemic events in the diltiazem group was not significant. However, diltiazem substantially decreased the overall number and the mean duration of transient ischemic events per patient, parameters that more accurately reflect the severity of the underlying ischemic process.

The results of perioperative changes in laboratory 

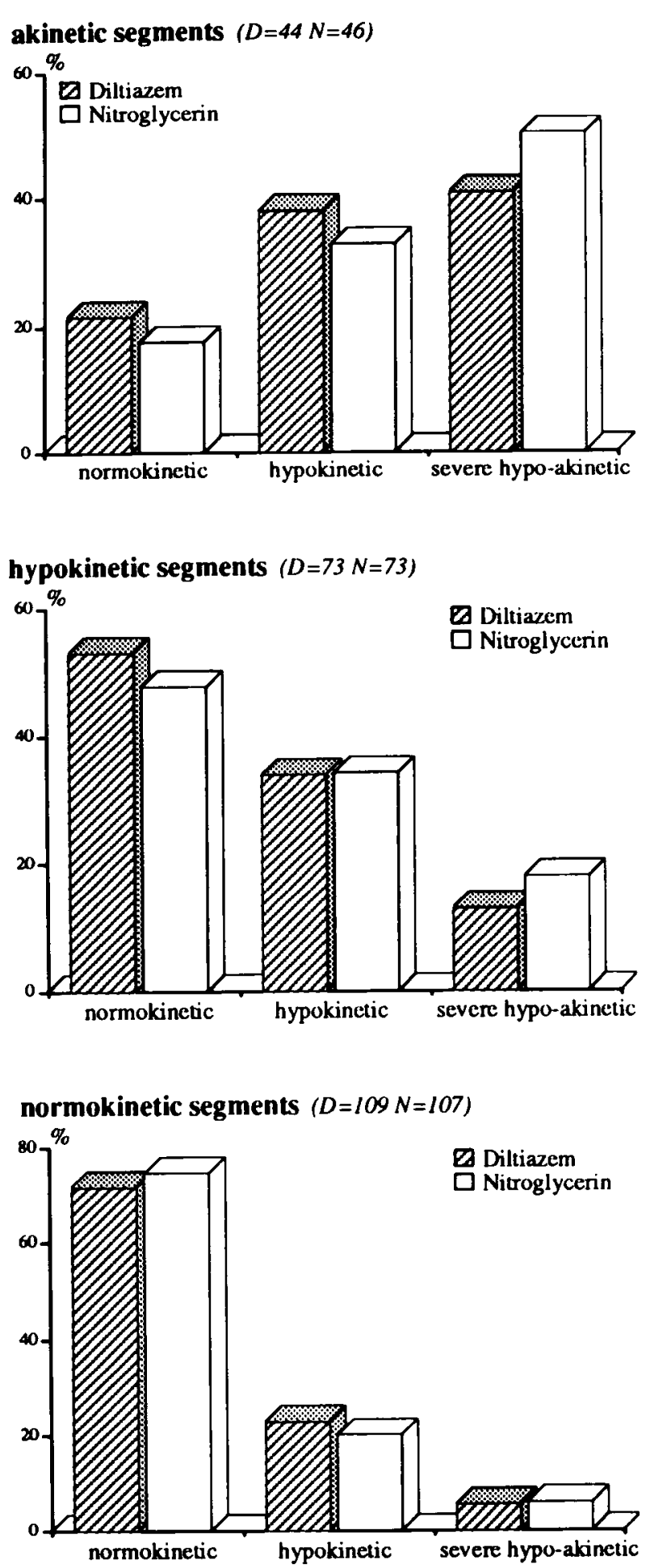

Fig. 2. Bar graph of changes in qualitatively graded regional systolic myocardial function (normal, hypokinetic, or severe hypokinetic-akinetic) at 4 hours after crossclamp removal. Data are given as percent change from preoperative values. $D$, Diltiazem; $N$, nitroglycerin.
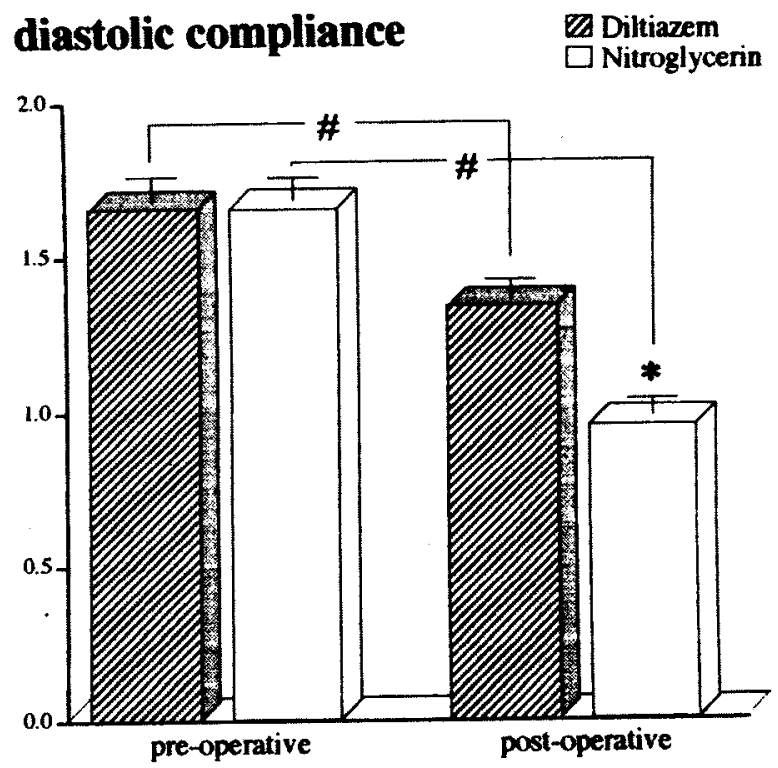

Fig. 3. Bar graph of changes in diastolic compliance (ratio of velocity time integrals during early and late diastolic filling). Data are given as mean \pm standard error of the mean. *Value differs significantly from that for diltiazem group $(p<0.05)$. \#Value differs significantly from the preoperative value $(p<$ $0.05)$.

parameters also demonstrated an antiischemic efficacy of diltiazem. Highly ischemia-specific laboratory parameters such as CK-MB-M and troponin- $T$ and also conventional parameters such as $\mathrm{CK}$ and $\mathrm{CK}-\mathrm{MB}$ were measured to accurately assess the extent of perioperative cell necrosis. Cardiac troponin-T, a polypeptide subunit of the myofibrillar regulatory troponin complex, is a myocardial antigen that is able to differentiate between skeletal and cardiac muscle damage with significantly greater specificity than by measurements of CK and CK-MB. ${ }^{25,26} \mathrm{~A}$ comparably high specificity for the detection of cardiac cell damage has been reported for the CK-MB-M analysis. ${ }^{27}$ These unique capabilities of troponin- $T$ and CK-MB-M appear to be of particular diagnostic importance in patients having CABG, in whom substantial skeletal muscle damage may occur during sternotomy or dissection of the internal mammary artery. ${ }^{25}$ In addition, the high specificity of troponin-T and CK-MB-M for even minor cardiac cell necrosis allows the identification of patients with non-Q-wave infarction as well as those with ST-segment alterations during periods of unstable angi$\mathrm{na}^{26}$

In the present study, peak values of CK-MB, CK-MB$M$, and troponin- $T$ were significantly lower in the diltiazem group. Troponin- $T$ levels in the diltiazem group were actually almost half of those observed in the nitroglycer- 
in group. In the subgroups of patients with either transient ischemic events or myocardial infarction, only the analysis of troponin-T and CK-MB-M allowed differentiation between the two treatment groups. Both parameters were substantially lower in diltiazem-treated patients, indicating that the extent of cardiac cell necrosis was significantly lower. In this respect, it may be concluded that the markedly lower numbers and shorter durations of transient ischemic events in the diltiazem group do reflect a clinically significant antiischemic effect.

Effects of diltiazem on myocardial function. Transesophageal echocardiography was used to investigate whether or not diltiazem significantly depresses postoperative myocardial function. Myocardial performance early after cardiopulmonary bypass depends on multiple factors such as preload and afterload, variations in heart rate, and level of anesthesia, which may complicate the assessment of direct drug-related effects. Therefore, we compared changes in myocardial function between periods of relative hemodynamic stability such as immediately before the operation and 4 hours after opening of the aortic clamp. Qualitative grading of segmental myocardial function is the most common method in perioperative monitoring by transesophageal echocardiography. ${ }^{28-31}$ However, this method is unable to detect small changes in regional wall motion and cannot be used to define global changes in the inotropic state of the myocardium. In contrast, the computer-assisted calculation of the fractional area of contraction (\%FAC) is a widely accepted quantitative method for assessing even small changes in global myocardial function. ${ }^{31,32}$ In addition, the measurement of changes in systolic wall thickening certainly provides the most precise method for quantitative analysis of changes in regional myocardial function. ${ }^{30,31,33}$

Quantitative assessment of perioperative changes in myocardial function revealed a significant improvement of global myocardial function (\%FAC) in the diltiazem group but not in the nitroglycerin group. The diltiazeminduced improvement in global function was probably caused by the observed augmentation of percent systolic thickening in the anterior wall. Our data cannot explain the slight but significant reduction of regional function in the posterior wall of patients treated with nitroglycerin. However, although there appears to be no rational explanation, it has been shown that postoperative regional wall motion abnormalities are more often located in the posterior than in the anterior wall. ${ }^{28}$ Because our Holter data do not allow the exact localization of ST-segment alterations, it remains unclear whether the higher prevalence and severity of perioperative ischemic events in the nitroglycerin group were mainly caused by ischemia in the posterior wall and may explain the significant reduction of percent systolic wall thickness in this area. The results of qualitative segmental grading support the findings of our quantitative measurements. Although there was a trend toward more postoperative normokinetic segments in both treatment groups, the number of severely hypokinetic segments was lower in the diltiazem group at postoperative evaluation. In addition, the postoperative improvement in functional grading was more pronounced in the diltiazem group in segments that showed severe hypokinetic-akinetic function before the operation. Although the differences were not significant, these trends indicate a beneficial effect of diltiazem on postoperative function and confirm the reported protective efficacy of diltiazem in segments of most severe dysfunction. ${ }^{34}$

Impaired relaxation of the left ventricle during diastole has been consistently observed in patients with coronary artery disease and often reflects or even precedes a deterioration in systolic function. ${ }^{35-37}$ In this study, diastolic compliance was characterized by the ratio between early diastolic and late atrial filling as assessed by transmitral diastolic Doppler flow velocity time integrals. This index has been shown to be a useful parameter in providing a noninvasive and reliable estimate of diastolic performance, especially in patients with coronary artery disease. $^{35,38}$ Preoperatively, the flow/velocity ratio was comparable in both groups (1.66 versus 1.68 ) and indicated a slightly decreased diastolic compliance when compared with values for patients with normal ventricular function. ${ }^{38}$ No comparable data exist to confirm our findings that at 4 hours after CABG diastolic function is significantly depressed. The observed postoperative decrease of the flow/velocity ratio in both groups apparently reflects the effect of intraoperative ischemia on the diastolic compliance. This assumption is substantiated by the fact that, except for heart rate, hemodynamic variables that may influence the assessment of diastolic function, such as systemic arterial, central venous, or pulmonary wedge pressure, were almost identical in both treatment groups. In this respect, the significantly lower reduction of the flow/velocity ratio in the diltiazem group indicates a beneficial effect of diltiazem on perioperative preservation of diastolic function.

\section{REFERENCES}

1. Akins CW, Block PC, Palacios IF, Gold HK, Carroll DL, Grunkemeier GL. Comparison of coronary artery bypass grafting and percutaneous transluminal coronary angioplasty as initial treatment strategies. Ann Thorac Surg 1989;47:507-16.

2. Daily PO. Early and five-year results for coronary artery bypass grafting. J ThORAC CARDIOvaSC SURG 1989;97: 67-77. 
3. Hochberg MS, Gielchinsky I, Parsonnet V, Hussain SM, Mirsky E, Fisch D. Coronary angioplasty versus coronary bypass: three-year follow-up of a matched series of 250 patients. J ThoraC CARdiovasc SuRg 1989;97:496-503.

4. Seitelberger R, Zwölfer W, Binder TM, et al. Infusion of nifedipine following coronary artery bypass grafting decreases the incidence of early postoperative myocardial ischemia. Ann Thorac Surg 1990;49:61-8.

5. Seitelberger R, Zwölfer M, Huber S, et al. Nifedipine reduces the incidence of myocardial infarction and transient ischemia in patients undergoing coronary bypass grafting. Circulation 1991;83:460-8.

6. Hannes W, Fasol R, Zajonc H, et al. Diltiazem provides anti-ischemic and anti-arrhythmic protection in patients undergoing coronary bypass grafting. Eur J Cardiothorac Surg 1993;7:239-45.

7. Chaffman M, Brodgen RN. Diltiazem: a review of its pharmacological properties and therapeutic efficacy. Drugs 1985;29:387-454.

8. Millard RW, Grupp G, Grupp IL, DiSalvo J, DePover A, Schwartz A. Chronotropic, inotropic, and vasodilator actions of diltiazem, nifedipine, and verapamil: a comparative study of physiological responses and membrane receptor activity. Circ Res 1983;52(Suppl I):29-39.

9. Podesser B, Schwarzacher S, Zwölfer W, et al. Combined perioperative infusion of nifedipine and metoprolol provides antiischemic and antiarrhythmic protection in patients undergoing elective aortocoronary bypass surgery. Thorac Cardiovasc Surg 1993;41:173-80.

10. Matsuzaki M, Gallagher KP, Patritti J, et al. Effects of calcium-entry blocker (diltiazem) on regional myocardial flow and function during exercise in conscious dogs. Circulation 1984;69:801-14.

11. Melendez FJ, Gharagozloo F, Sun SC, et al. Effects of diltiazem cardioplegia on global function, segmental contractility, and the area of necrosis after acute coronary artery occlusion and surgical reperfusion. J THORAC CARDIOVASC SURG 1988;95:613-7.

12. Petru MA, Crawford MH, Sorensen SG, Chaudhuri TK, Levine S, O'Rourke RA. Short and long-term efficacy of high-dose oral diltiazem for angina due to coronary artery disease: a placebo-controlled, randomized, double-blind crossover study. Circulation 1983;68:139-47.

13. Ball RM, Bache RJ. Distribution of myocardial blood flow in the exercising dog with restricted coronary inflow. Circ Res 1976;38:60-6.

14. Slogoff S, Keats AS. Does chronic treatment with calcium entry blocking drugs reduce perioperative myocardial ischemia? Anesthesiology 1988;68:676-80.

15. Gülker H, Ketteler TH. Antiarrhythmische Wirkungen der Kalzium-antagonisten. Z Kardiol 1989;78(Suppl):49-54.

16. Rozanski JJ, Zaman L, Castellanos A. Electrophysiologic effects of diltiazem hydrochloride on supraventricular tachycardia. Am J Cardiol 1982;49:621-8.

17. Sternbach GL, Schroeder JS, Eliastam M, Beier-Scott L. Intravenous diltiazem for the treatment of supraventricular tachycardia. Clin Cardiol 1986;9:145-9.
18. Talajic M, Papadatos D, Villemaire C, Nayebpour $M$, Nattel S. Antiarrhythmic actions of diltiazem during experimental atrioventricular reetrant tachycardias: importance of use-dependent calcium channel-blocking properties. Circulation 1990;81:334-42.

19. Michelson EL, Morganroth J, MacVaugh H. Postoperative arrhythmias after coronary artery and cardiac valvular surgery detected by long-term electrocardiographic monitoring. Am Heart J 1979;97:442-8.

20. Henling CE, Slogoff S, Kodali V, Arlund C. Heart block after coronary artery bypass: effect of chronic administration of calcium-entry blockers and $\beta$-blockers. Anesth Analg 1984;63:515-20.

21. Woelfel A, Foster JR, Simpson RJ, Gettes LS. Reproducibility and treatment of exercise induced ventricular tachycardia. Am J Cardiol 1984;53:751-6.

22. Goldberger AL. Normal and noninfarct $Q$ waves. Cardiol Clinics 1987;5:357-66.

23. Raabe DS Jr, Morise A, Sbarbaro JA, Gundel WD. Diagnostic criteria for acute myocardial infarction in patients undergoing coronary artery bypass surgery. Circulation 1980;62:869-78.

24. Hannes W, Zajonc $H$, Krause $T$, et al. Wertigkeit verschiedener diagnostischer Methoden zur Erfassung der perioperativen Myokardnekrose nach aortokoronarer Bypassoperation. Schweiz Med Wochenschr 1993;12(Suppl 49):P123.

25. Katus HA, Schoeppenthau M, Tanzeem A, et al. Non-invasive assessment of perioperative myocardial cell damage by circulating cardiac troponin T. Br Heart J 1991;65:25964.

26. Katus HA, Remppis A, Neumann FJ, et al. Diagnostic efficiency of troponin $\mathrm{T}$ measurements in acute myocardial infarction. Circulation 1991;83:902-12.

27. Gerhardt W, Katus H, Ravkilde J, et al. S-troponin $T$ in suspected ischemic myocardial injury compared with mass and catalytic concentrations of S-creatine kinase isoenzyme MB. Clin Chem 1991;37:1405-11.

28. Leung JM, O'Kelly B, Browner WS, Tubau J, Hollenberg M, Mangano DT. Prognostic importance of postbypass regional wall motion abnormalities in patients undergoing coronary artery bypass graft surgery. Anesthesiology 1989; 71:16-25.

29. Poelaert JIT, Reichert CLA, Koolen JJ, Everaert JA, Visser CA. Transesophageal echo-Doppler evaluation of the hemodynamic effects of positive-pressure ventilation after coronary artery surgery. J Cardiothorac Vasc Anesth 1992;6:438-43.

30. Topol EJ, Weiss JL, Guzman PA, et al. Immediate improvement of dysfunctional myocardial segments after coronary revascularization: detection by intraoperative transesophageal echocardiography. J Am Coll Cardiol 1984;4:1123-34.

31. Rosenthal A, Kawasuji M, Takemura H, Sawa S, Iwa T. Transesophageal echocardiographic monitoring during coronary artery bypass surgery. Jpn Circ J 1991;55:109-16.

32. Schnittger I, Fitzgerald PJ, Gordon EP, Alderman EL, 
Popp RL. Computerized quantitative analysis of left ventricular wall motion by two-dimensional echocardiography. Circulation 1984;2:242-54.

33. Ren JF, Kotler MN, Hamid Hakki A, Panidis IP, Mintz GS, Ross J. Quantitation of regional left ventricular function by two-dimensional echocardiography in normals and patients with coronary artery disease. Am Heart J 1985;110:552-60.

34. Taylor AL, Golino P, Eckels R, Pastor P, Buja M, Willerson JT. Differential enhancement of postischemic segmental systolic thickening by diltiazem. J Am Coll Cardiol 1990;15:737-47.

35. Appleton CP, Hatle LK, Popp RL. Relation of transmitral flow velocity patterns to left ventricular diastolic function: new insights from a combined hemodynamic and Doppler echocardiographic study. J Am Coll Cardiol 1988;12:42640.

36. Aroesty JM, McKay RG, Heller GV, Royal HD, Als AV, Grossman W. Simultaneous assessment of left ventricular systolic and diastolic dysfunction during pacing-induced ischemia. Circulation 1985;71:889-900.

37. Courtois M, Mechem CJ, Barzilai B, Ludbrook PA. Factors related to end-systolic volume are important determinants of peak early diastolic transmitral flow velocity. $\mathrm{Cir}-$ culation 1992;85:1132-8.

38. Spirito P, Maron BJ, Bonow RO. Noninvasive assessment of left ventricular diastolic function: comparative analysis of Doppler echocardiographic and radionuclide angiographic techniques. J Am Coll Cardiol 1986;7:518-26.

\section{Discussion}

Dr. Charles A. Dietl (Danville, Pa.). Dr. Seitelberger and his colleagues have brought to our attention the usefulness of calcium channel blockers in the perioperative period.

Professor Carpentier advocated the administration of 0.1 $\mathrm{mg} / \mathrm{kg}$ per hour of intravenous diltiazem followed by 180 to 240 $\mathrm{mg}$ of oral diltiazem daily for several months after discharge to prevent spasm in all patients with radial artery grafts. In November of 1992, we introduced simultaneously in our practice the routine administration of intravenous and oral diltiazem and the radial artery conduit.

Our small experience includes 37 patients treated according to the protocol recommended by Professor Carpentier from a total of 98 patients in whom two, three, or four different types of arterial conduits were used, including 128 internal mammary artery grafts, 60 internal epigastric artery grafts, 38 radial artery grafts, and 23 gastroepiploic artery grafts. This group study includes 49 patients in whom saphenous vein grafts were used concomitantly. Interestingly, I did not notice any intraoperative spasm in any radial artery graft, whereas the other arterial conduits, especially the gastroepiploic artery, seem to be more spasmogenic. There was no statistical difference in mortality in the group with or without diltiazem ( $2.7 \%$ versus $3.2 \%$ ), and these deaths occurred in patients with severely impaired ventricular function.

In the group treated with calcium channel blockers, two patients had transient ischemic changes in the electrocardio- gram. No Q-wave perioperative myocardial infarctions occurred, although the echocardiogram showed inferior wall hypokinesis in both patients, and all patients were free of symptoms. However, among 61 patients not treated with diltiazem, two patients $(3.2 \%)$ had a $Q$-wave perioperative infarction and two patients had recurrent angina. Ventricular arrhythmias were somewhat more prevalent in the nontreated group as well.

Our data suggest that diltiazem may be beneficial during the perioperative period in all patients with different types of arterial conduits for revascularization. I would like to know what experience the author has with other types of arterial conduits.

Dr. James L. Cox (St. Louis, Mo.). I notice that you administered diltiazem and used Holter monitors for 24 hours after the operation. Since atrial fibrillation occurs usually 2 to 4 days postoperatively, have you any information as to why this should decrease the prevalence of postoperative atrial fibrillation? Second, did the postoperative improvement in the diastolic compliance of the ventricles persist after cessation of the drug?

Dr. Seitelberger. Thank you very much for your comments. With regard to our experience with various arterial conduits, I can only say that in this study one or two mammary arteries were used in $85 \%$ of both treatment groups. However, we did not use other arterial conduits such as the radial artery, as reported by Dr. Carpentier. From our data it is difficult to judge the effect of diltiazem on the prevalence of postoperative spasms in arterial conduits. However, we observed that the most significant increase in postoperative myocardial function was located in the anterolateral wall, which was the area usually revascularized with the mammary artery. Although we cannot answer this question conclusively, we suppose that the vasodilatory efficacy of diltiazem also increased the flow through the mammary artery, which was present in almost every patient. Consequently, this effect may explain the differences in postoperative function in the anterolateral wall of patients with an arterial conduit in the diltiazem group when compared with the nitroglycerin group.

With regard to the prevalence of postoperative atrial fibrillation, I have to mention that many patients who received the diltiazem infusion throughout the perioperative period were then given further, peroral diltiazem medication. I think this was one of the major reasons that atrial fibrillation was significantly less prevalent in the diltiazem group. However, since we also saw a relatively high incidence of atrial fibrillation early after the operations, which is normally only transient and can only be detected by Holter monitoring, we were also able to detect the antiarrhythmic efficacy of diltiazem in the early postoperative period.

With regard to perioperative changes in the diastolic compliance of the left ventricle, it was very difficult for us to find any report in the literature concerning this problem. Therefore, we cannot compare our results with those of any other study. In our study we evaluated the diastolic compliance only before the operation and at 4 hours thereafter. I cannot comment on any further changes in the diastolic compliance after this period, such as the first 5 postoperative days. Because no comparable data exist in the literature, we can only speculate that our data do reflect the typical course of changes in diastolic compliance in the early postoperative period. In this respect, our results show a protective effect of diltiazem on diastolic compliance, but we cannot say whether this protective effect also holds true for a longer period after the operation. 